$$
\text { ANL/PHY/CP-95025 }
$$

\title{
First Use of a Laser-Driven Polarized H/D Target at the IUCF Cooler
}

\author{
M. A. Miller ${ }^{1 a}$, K. Bailey ${ }^{b}$, J. Brack $^{c}$, R. V. Cadman ${ }^{a}$, \\ W. J. Cummings ${ }^{b}$, J. Fedchak ${ }^{2 b}$, B. Fox ${ }^{c}, \mathrm{H} . \mathrm{Gao}^{3 b}$, \\ C. Grosshauser ${ }^{e}$, R. J. Holt ${ }^{a}$, C. Jones ${ }^{g}$, E. Kinney ${ }^{c}$, \\ R. Kowalczyk ${ }^{b}$, Z.-T. Lu ${ }^{b}$, W. Nagengast ${ }^{e}$, B. Owen ${ }^{a}$, \\ K. Rith ${ }^{e}$, F. Schmidt ${ }^{e}$, E. Schulte ${ }^{a}$, J. Sowinski $^{f}$, \\ F. Sperisen ${ }^{f}$, J. Stenger ${ }^{4}$, E. Thorsland ${ }^{a}$, \\ and S. Williamson ${ }^{a}$ \\ ${ }^{a}$ University of Ilinois at Urbana-Champaign, Urbana, Mlinois 61801 \\ ${ }^{b}$ Argonne National Laboratory, Argonne, Illinois 60439 \\ ${ }^{c}$ University of Colorado at Bolder, Bolder, Colorado 80309-0446 \\ ' Universität Erlangen-Nürnberg, 91058 Erlangen, Germany \\ ${ }^{a}$ Massachusetts Institute of Technology, Cambridge, Massachusetts 02139 \\ Indiana University Cyclotron Facility, Bloomington, Indiana 47408 \\ ${ }^{g}$ California Institute of Technology, Pasadena, California 91125
}

\begin{abstract}
The HERMES Laser-Driven Target Task Force (Argonne, Erlangen and Illinois) is charged with developing a polarized H/D target for use in the HERA ring at DESY. Rapid progress was made in the beginning of 1996, leading us to the decision to test the target in a realistic experimental environment. In particular, polarizations of 0.6 and flows above $10^{18}$ atoms $\cdot \mathrm{s}^{-1}$ have been achieved on the bench. The laser-driven target and a simple detector system are currently installed in Cooler storage ring at the Indiana University Cyclotron Facility in order to test its applicability to nuclear physics experiments. Target polarizations are being measured using the $\vec{H}(p, p)$ and $\vec{D}(p, p)$ reactions. Initial tests were reasonably successful and the target is well along toward becoming viable for nuclear physics
\end{abstract}

1) Corresponding author, miller5@uiuc.edu

2) Current address: University of Wisconsin-Madison, Madison, Wisconsin

3) Current address: Laboratory for Nuclear Science, Massachusetts Institute of Technology, Cambridge, MA 02139

4) Current address: Massachusetts Institute of Technology, Cambridge, MA 02139 


\section{DISCLAIMER}

This report was prepared as an account of work sponsored by an agency of the United States Government. Neither the United States Government nor any agency thereof, nor any of their employees, make any warranty, express or implied, or assumes any legal liability or responsibility for the accuracy, completeness, or usefulness of any information, apparatus, product, or process disclosed, or represents that its use would not infringe privately owned rights. Reference herein to any specific commercial product, process, or service by trade name, trademark, manufacturer, or otherwise does not necessarily constitute or imply its endorsement, recommendation, or favoring by the United States Government or any agency thereof. The views and opinions of authors expressed herein do not necessarily state or reflect those of the United States Government or any agency thereof. 


\section{DISCLAIMER}

Portions of this document may be illegible in electronic image products. Images are produced from the best available original document. 


\section{INTRODUCTION}

Polarized targets are an important tool for nuclear physics, used both for investigating spin-dependence in nuclear reactions and structure and for studying the spin structure of the nucleon. The laser-driven polarized hydrogen and deuterium target, originally described in Ref. 1, has been developed to provide a high density polarized gas target for storage ring experiments. Since that time, development has continued at several laboratories ${ }^{5}$ and has reached a point where the target is ready to be tested in a realistic nuclear physics environment.

In order for a target to be useful for nuclear physics, several criteria have to be met. The target thickness has to be sufficient to provide high enough luminosity to carry out an experiment, the nuclear polarization also must be high enough to provide a measurable spin-dependent signal and the target must be robust enough to maintain thickness and polarization continuously over the days, weeks, or even months, of an experiment. The laser-driven source has an advantage in this first area. Typical operating flows are $10^{18}$ nuclei/second. At present, the limitation on flow comes from the affect of the target on the lifetime of a stored beam in the IUCF ring, rather than from the source itself.

The nuclear polarization in the source is produced in several steps. Atoms are polarized by spin exchange collisions between $\mathrm{D}$ (or $\mathrm{H}$ ) atoms, produced by an RF dissociator, and polarized potassium atoms. A small fraction of potassium atoms is introduced into the source from a potassium ampoule which is heated to provide the desired density. The potassium atoms are optically pumped by polarized $770.1 \mathrm{~nm}$ laser light from standing-wave Ti-sapphire lasers pumped by two $\mathrm{Ar}^{+}$lasers. Polarization is transfered from the $\mathrm{K}$ atoms to H/D atoms in spin-exchange collisions and then to H/D nuclei by $\mathrm{HH}$ or DD spin-exchange collisions which bring the system to spin-temperature equilibrium, as discussed elsewhere in this proceedings [7] and in Ref. 8. In order to maintain the polarization of the nuclei in the source and as they move to the target, the source is coated with drifilm [9], which minimizes recombination into molecules and depolarization. Atomic polarizations of $p_{e} \sim 0.6$ have been achieved with atomic fraction $\sim 0.5$. Typical polarization behavior on the bench is shown in Fig. 1, with $p_{z}=0.6$, and an atomic fraction of 0.35 . The source is described in more detail in Refs. 1-5 and elsewhere in these proceedings [6].

The effective nuclear polarization of the target, $p_{\text {eff }}$, depends several quantities: the electronic polarization of the atoms, the atomic fraction, whether the

5) The HERMES laser-driven target task force consists of research groups at the University of Illinois at Urbana-Champaign, Argonne National Laboratory, the Universität ErlangenNürnberg and the University of Colorado at Boulder. 


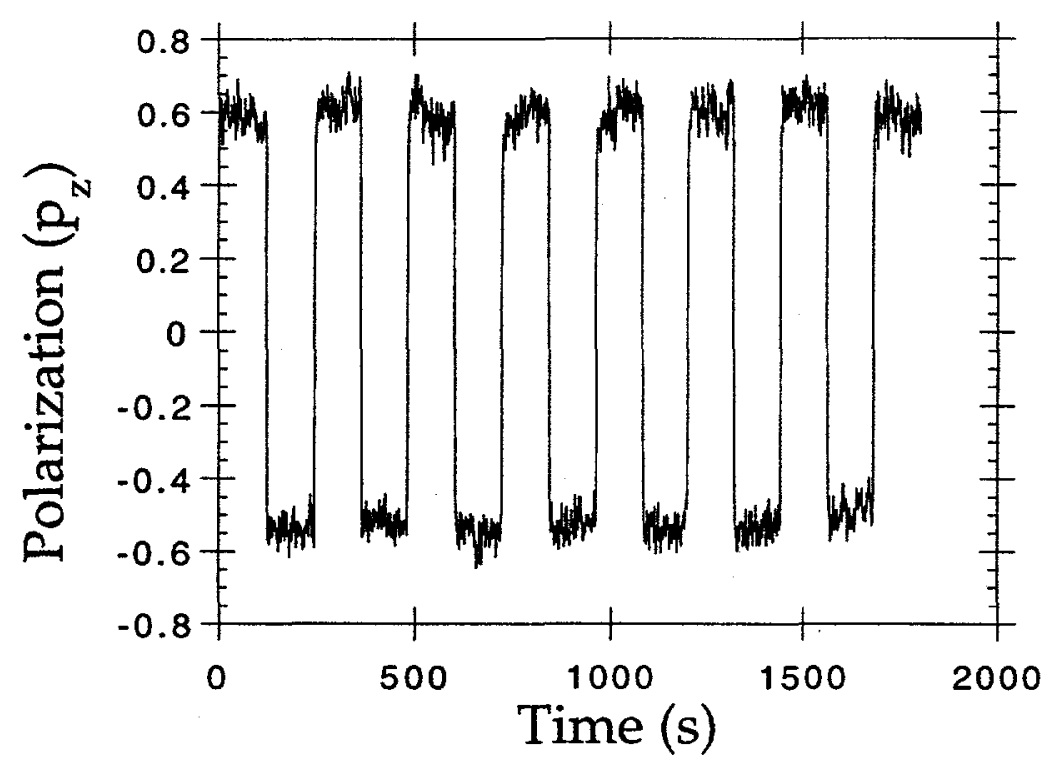

FIGURE 1. Polarization performance of the laser-driven source. This is the polarization $p_{z}$ of deuterons, assuming that the system is in spin temperature equilibrium, plotted versus time. The sign of the polarization is changed by switching the helicity of the laser light incident on the target.

system has achieved spin-temperature equilibrium and depolarization caused in the source (from collisions with surfaces and gas, from recombination effects). In addition, if the target is used in an experiment which probes the nucleon spin (for example, deep-inelastic electron scattering), the largely unpolarized nucleons in small fraction of the potassium atoms in the target dilutes the overall nuclear polarization (typical potassium densities are $\sim 0.1 \%$ of the $\mathrm{H} / \mathrm{D}$ density). It should be noted that this assumes that nuclei in atoms that have recombined into molecules are depolarized in the process. If these nuclei retain some or all of their original polarization, the effective polarization would be significantly larger than that predicted from the atomic fraction.

\section{INSTALLATION IN THE COOLER}

The Cooler light ion storage ring $[10,11]$ at the Indiana University Cyclotron Facility (IUCF) is well suited to demonstrate that the target is viable under realistic experimental conditions. The main goals of this project are to install the target in the Cooler, to measure the nuclear polarization of the target and to measure the polarization of nuclei in molecules. After this program is 


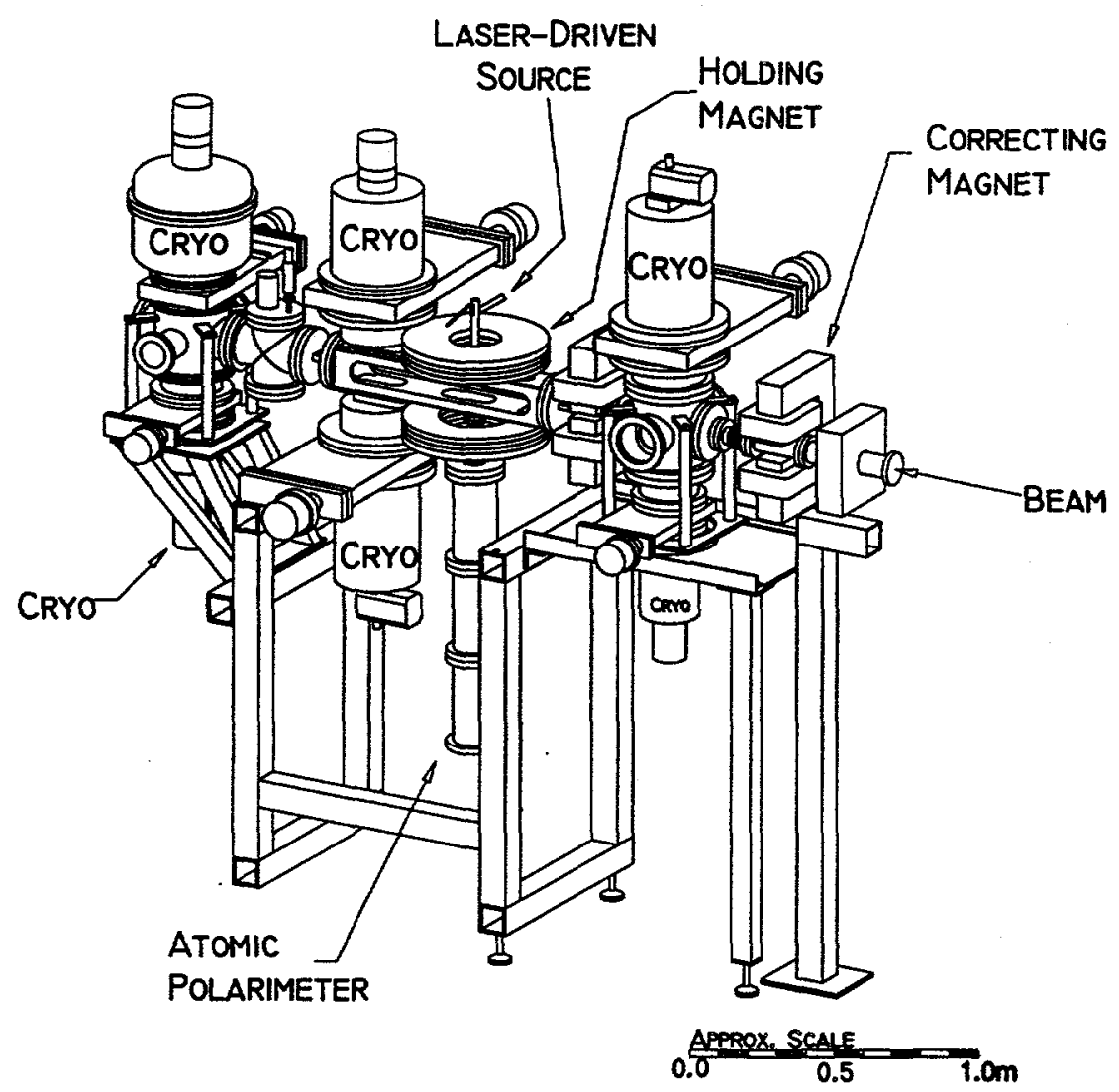

FIGURE 2. A view of the polarized internal $H / D$ target and beam line as installed in the Cooler G-region. The Cooler beam passes from right to left in the figure. The two correcting magnets compensate for the deflection of the beam due to the target holding field.

complete, the target will be used study the spin-dependence of the deuterium wave function via the $\vec{D}(\vec{p}, 2 p)$ and $\vec{D}(\vec{p}, p n)$ reactions. The target installation in the Cooler G-region is shown in Fig. 2.

The target cell dimensions and the flux of polarized nuclei from the source define the target thickness. In the present experiment, the horizontal and vertical dimensions of the target cell are chosen such that the phase space acceptance of the target nearly matches the design acceptance of the accelerator. The target cell is made from a single piece of aluminum $400 \mathrm{~mm}$ long, $32 \mathrm{~mm}$ wide by $13 \mathrm{~mm}$ high. Its sides have been machined to provide exit windows approximately $0.2 \mathrm{~mm}$ thick. The ends of the cell are open to allow the Cooler beam to pass through. The polarized source is mounted above the target cell with a vertical polarization axis defined by a vertical holding field $\sim 1 \mathrm{kG}$. The inner surfaces of both the pumping cell and the target cell are coated with drifilm to minimize the wall depolarization and recombination 


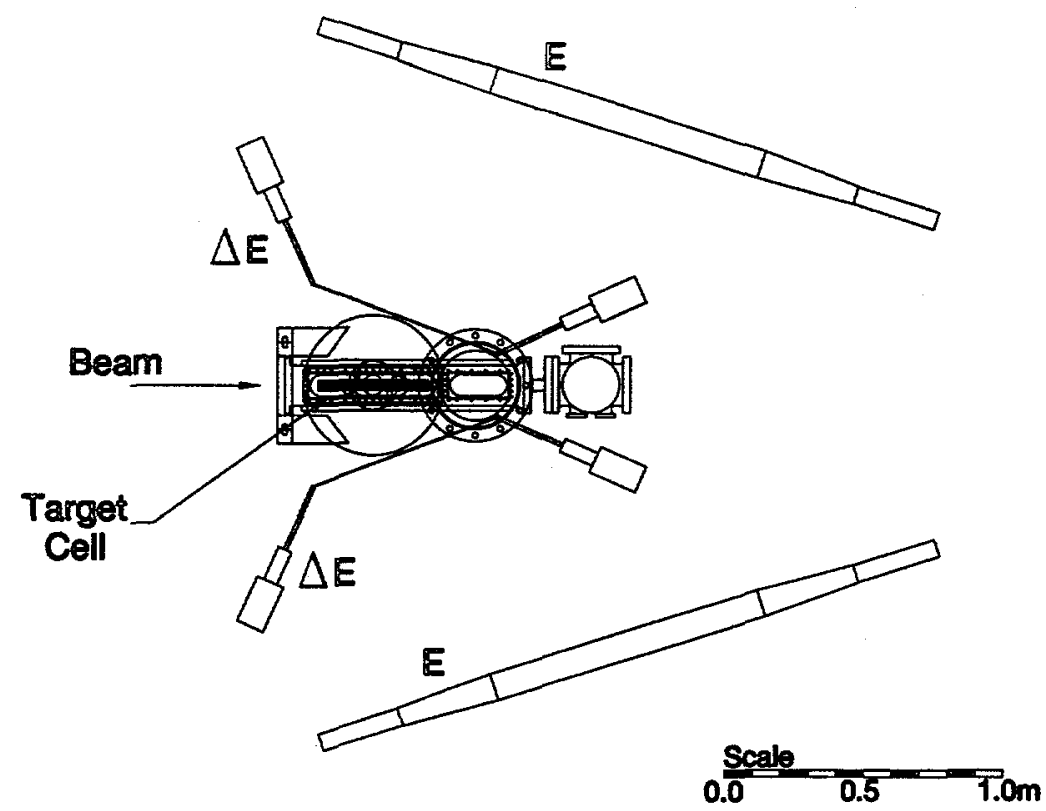

FIGURE 3. An overhead view of the target and detector arrangement used to measure the target polarization. The $200 \mathrm{MeV}$ polarized proton beam enters from the left. $\Delta \mathrm{E}$ and E scintillator detectors are placed symmetrically on either side of the target.

effects. The source and target are heated to $\sim 250 \mathrm{C}^{\circ}$ to prevent potassium atoms from condensing onto the wall surfaces.

The detector arrangement, shown in Fig. 3, consists of thin plastic scintillators (one on either side of the target, $3 \mathrm{~mm}$ thick) and scintillator bars (two per side, $1 \mathrm{~m}$ long by $100 \mathrm{~mm}$ thick and $150 \mathrm{~mm}$ high) acting as $\Delta \mathrm{E}-\mathrm{E}$ pairs The extended target and large detectors provide coverage for approximately $40-100^{\circ}$ in the $\mathrm{p}+\mathrm{p}$ center of mass. Particle identification provided by timeof-flight between $\Delta \mathrm{E}$ and $\mathrm{E}$ and $d E / d x$ allows the separation of protons and deuterons. Time differences between photo-tubes at each end of the $\triangle E$ and $\mathrm{E}$ detectors are used to measure the angles of detected particles. A coincidence between both $\Delta \mathrm{E}$ detectors and at least one $\mathrm{E}$ detector is required for a trigger.

The laser system for optical pumping of the source consists of two $\mathrm{Ar}^{+} / \mathrm{Ti}^{-}$ sapphire pairs with the $\mathrm{Ar}^{+}$lasers acting as pump lasers for the tunable $\mathrm{Ti}$ sapphire lasers. They are installed outside the Cooler approximately $60 \mathrm{~m}$ from the target area with the laser light transported to the target on multimode fiber optics, as described elsewhere in this proceedings [12]. This fiber optic transport allows us to place the lasers in an easily accessible location where they can be tuned while the Cooler is operating. The net efficiency of the light transport is $\sim 70 \%$, with the losses dominated by input and output coupling to the fibers. The light is split into two polarization states at the 
target and recombined after the polarizations have been rotated so that all of the light is circularly polarized. The helicity can be switched by changing between two $\lambda / 4$ plates in a sliding stage.

An atomic beam polarimeter, consisting of a permanent quadrupole separator magnet and mass spectrometer is installed beneath the target chamber. This is used to measure the polarization and atomic fraction of the atoms coming from the source. A second mass spectrometer (the dissometer) looks at the downstream end of the target cell through a series of apertures. This is used to measure the atomic fraction of atoms coming out of the end of the target cell. The combination of these two atomic fraction measurements, at the center and at the end of the target cell, gives us an important diagnostic of recombination inside the target cell. In addition to the pump lasers, two beams from diode probe lasers are brought from the laser room to the target on separate fibers. These are used to measure relative potassium density in the source (through photo-absorption) and the atomic polarization of the potassium (via Faraday rotation [13]).

\section{PRELIMINARY POLARIZATION MEASUREMENTS}

The elastic $\vec{H}(p, 2 p)$ and $\vec{D}(p, p d)$ reactions are used to measure the polarizations of the target when running hydrogen and deuterium, respectively. The reactions have relatively large analyzing powers $\left(A_{n 0} \sim 0.3\right.$ at $\theta_{c m} \sim 40^{\circ}$ for $\overrightarrow{\mathrm{H}}(\mathrm{p}, 2 \mathrm{p})$ and $i t_{11} \sim 0.35$ at $\theta_{c m} \sim 40^{\circ}$ for $\left.\overrightarrow{\mathrm{D}}(\mathrm{p}, \mathrm{pd})\right)$ and differential cross sections of a few $\mathrm{mb} / \mathrm{sr}$. This allows us to measure the asymmetries with a relative uncertainty of a few percent in 15 minutes or so.

We have run the target with beam twice to date. The measured spin asymmetry for the $\overrightarrow{\mathrm{H}}(\mathrm{p}, 2 \mathrm{p})$ reaction with the target both polarized and unpolarized is plotted versus center of mass scattering angle in Fig. 4. The running conditions here were not the best - the atomic fraction as measured by the atomic polarimeter was 0.50 while that measured with the dissometer was consistent with zero. This was a consequence of potassium migrating from the source to the target cell and damaging the drifilm coating. The curve shown in Fig. 4 is a fit of the known $\vec{H}(p, 2 p)$ analyzing power [14] to the data with a polarization parameter $p_{\text {eff }}$. The value of $p_{\text {eff }}$ is $0.09 \pm 0.01$, consistent with the assumption of a linearly decreasing atomic fraction, falling from 0.5 at the target center to zero at the ends of the target cell.

As mentioned above, the migration of potassium from the source is a concern with the LDS. In our first measurements at IUCF, potassium contaminated the target cell (probably due to an argon bubble formed in the ampoule in assembly), resulting in high recombination in the cell. Such problems will be avoided in the future by distilling the potassium before use. The potassium can also affect the dissociator, resulting in a gradual degradation of atomic fraction. Adding a small fraction of oxygen to H/D dissociators has long 
been known to improve dissociator lifetime and efficiency. Studies begun to investigate the affect of oxygen on the LDS dissociator have yielded promising initial results.

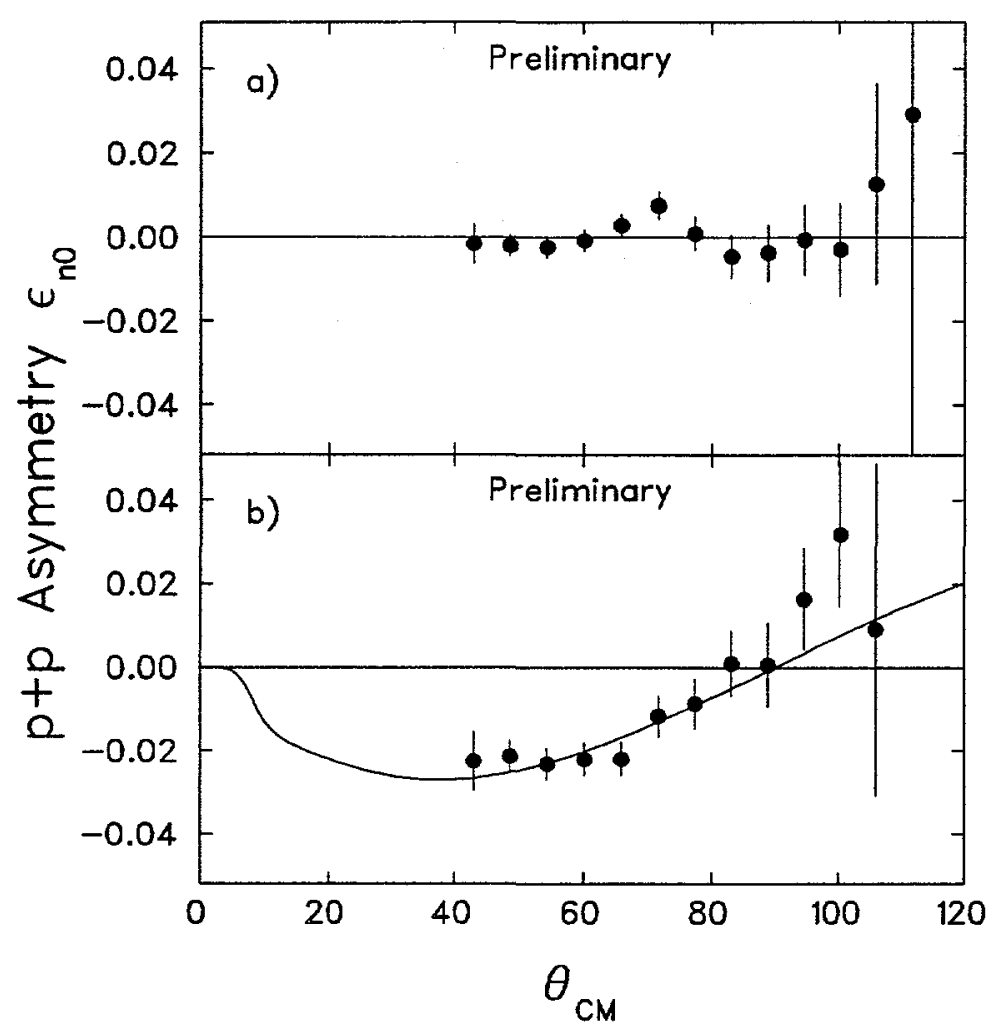

FIGURE 4. Preliminary measurements of the $\overrightarrow{\mathrm{H}}(\mathrm{p}, \mathrm{p})$ nuclear asymmetry $\left(\epsilon_{n 0}=p_{\text {eff }} A_{n 0}\right)$ plotted versus center of mass angle: a) the measured asymmetry with the target unpolarized, b) the asymmetry with the target polarized. The curve in $b$ is a fit of the $\vec{H}(p, p)$ analyzing power [14] to the data. The atomic fraction in the target cell is undetermined for this measurement, but varied between 0.5 and 0.0 . The running conditions and polarization are discussed in the text.

\section{SUMMARY}

A laser-driven polarized H/D target has been installed in the IUCF Cooler. First tests with proton beam have been started in order to measure the nuclear polarization of the target. The first measurements have shown that the principle of the target is sound and that it produces polarized nuclei. A number of areas have been identified where further improvements can be made. Over the coming months, further studies will be carried out in order to improve reliability and performance of the source and target. The target will be also used 
to study the spin-dependence of the deuteron wave function via the $\vec{D}(\vec{p}, 2 p)$ reaction (IUCF experiment CE68). The coupling of the high flux LDS and the high cross sections of the $(p, 2 p)$ reaction will allow us to measure the effective polarization of the nucleons in the deuteron for momenta to $350 \mathrm{MeV} / \mathrm{c}$.

\section{ACKNOWLEDGEMENTS}

The authors would like to thank D. Tupa for the loan of two Ti-sapphire lasers, W. Lawrence, our glass blower at UIUC, A. Kenyon for his help with assembly and maintenance of the target, J. Doskow for maintaining the vacuum system and the rest of the IUCF operators and staff for their assistance. This work was supported in part by the U.S. National Science Foundation under grants PHY94-20787 and PHY94-20470 and by the U.S. Department of Energy under contract No. W-31-109-ENG-38 and grant DE-FG02-86ER40269.

\section{REFERENCES}

1. K. P. Coulter et al., Phys. Rev. Lett. 68, 174 (1992).

2. M. Poelker et al., Phys. Rev. A 50, 2450 (1994).

3. H. Gao, R. V. Cadman, R. J. Holt, and E. Thorsland, in Proceedings of the International Workshop on Polarized Beams and Polarized Gas Targets, edited by H. P. gen. Scheick and L. Sydow (World Scientific, Singapore, 1996), p. 67.

4. M. Poelker et al., Nucl. Instrum. Meth. A 364, 58 (1995).

5. B. Owen et al., in Spin 96 Proceedings, edited by C. de Jager et al. (World Scientific, Singapore, 1996), pp. 490-494.

6. R. V. Cadman et al., in Proceedings of the Seventh International Workshop on Polarized Gas Targets and Polarized Beams, edited by R. J. Holt (American Institute of Physics, New York, 1997), in preparation.

7. J. Stenger et al., in Proceedings of the Seventh International Workshop on Polarized Gas Targets and Polarized Beams, edited by R. J. Holt (American Institute of Physics, New York, 1997), in preparation.

8. T. G. Walker and W. Happer, Rev. Mod. Phys. 69, 629 (1997).

9. D. R. Swenson and L. W. Anderson, Nucl. Instrum. Meth. B 29, 627 (1988).

10. H. O. Meyer, Indiana Cooler User Guide, 2nd ed., 1988.

11. R. E. Pollock, Annu. Rev. Nucl. Part. Sci. 41, 357 (1991).

12. W. Cummings et al., in Proceedings of the Seventh International Workshop on Polarized Gas Targets and Polarized Beams, edited by R. J. Holt (American Institute of Physics, New York, 1997), in preparation.

13. J. Stenger, M. Beckman, W. Nagengast, and K. Rith, Nucl. Instrum. Meth. A 384, 333 (1997).

14. R. Arndt and R. Workman, The SAID program: A Guide for Users, 1994, available through anonymous ftp at $\mathrm{ftp}: / / \mathrm{clsaid}$.phys.vt.edu/pub/said/ said_manual. 